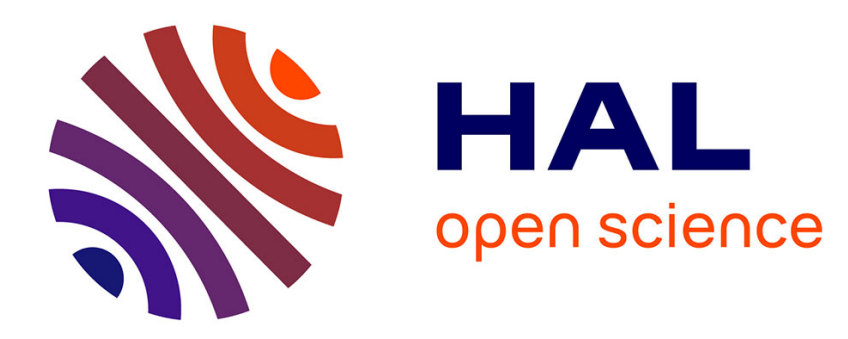

\title{
Le chèque comme outil du Nouveau Management Public dans les collectivités locales françaises
}

\author{
Arnaud Lacheret
}

\section{To cite this version:}

Arnaud Lacheret. Le chèque comme outil du Nouveau Management Public dans les collectivités locales françaises. Gestion et management public [2012-..], 2015, L'outil de gestion au service du New Public Management, 4 (1), pp.81-100. halshs-01276769

\section{HAL Id: halshs-01276769 \\ https://shs.hal.science/halshs-01276769}

Submitted on 20 Feb 2016

HAL is a multi-disciplinary open access archive for the deposit and dissemination of scientific research documents, whether they are published or not. The documents may come from teaching and research institutions in France or abroad, or from public or private research centers.
L'archive ouverte pluridisciplinaire HAL, est destinée au dépôt et à la diffusion de documents scientifiques de niveau recherche, publiés ou non, émanant des établissements d'enseignement et de recherche français ou étrangers, des laboratoires publics ou privés. 


\title{
Le chèque comme outil du Nouveau Management Public dans les collectivités locales françaises
}

\author{
Voucher as a tool of New Public Management in French local \\ governments
}

\author{
Arnaud Lacheret ${ }^{1}$
}

\begin{abstract}
Résumé: L'apparition et la diffusion des chèques comme outils de ciblage des aides dans les collectivités locales permet de faire un lien avec la notion de "vouchers » inventés dans les années 60 par Milton Friedman. Présentés comme neutre politiquement par les acteurs, la recherche a permis d'établir cette parenté avec ces outils du Nouveau Management Public dotés outre Atlantique d'une image très libérale. Ces outils se sont cependant diffusés sans heurts particuliers en France, notre étude s'est efforcée d'expliquer cette apparition dans un silence idéologique assourdissant.
\end{abstract}

Mots Clés : Chèque, ciblage, politique sociale, Nouveau Management Public, néolibéralisme

\begin{abstract}
The arrival and diffusion of vouchers as tools of targeting of public subsidies in french local government is linked with the notions of "vouchers" invented in the 1960s by Milton Friedman. Shown as neutral tools by their French users (politicians and civil servants), the research has made clearer the parenthood with between the vouchers of the two sides of the Atlantic as tools of New Public Management which are endowed with a very liberal image. These tools have spread among the French local governments without any debates. The present study has tried to explain this appearance within a great silence.
\end{abstract}

Key-words: Vouchers,targeting, social subsidies, New Public Management, neoliberalism

\footnotetext{
${ }^{1}$ Arnaud Lacheret est Docteur en Science Politique, il est chercheur associé au laboratoire Pacte/Science Po Grenoble et enseignant-chercheur à I'Idrac Business School. arnaud.lacheret@hotmail.fr
} 


\title{
Le chèque comme outil du Nouveau Management Public dans les collectivités locales françaises
}

\author{
Arnaud Lacheret, Pacte - Idrac research
}

La catégorisation des publics dans le recours à l'aide sociale renvoie à une notion ancienne mais qui semble n'avoir pas été beaucoup étudiée en France, le ciblage des aides ou " targeting ». Notre pays n'a en effet pas fait exception à la tendance mondiale visant à cibler les aides sociales en utilisant souvent un instrument particulièrement connoté: le chèque (voucher, dans la littérature internationale).

Le ciblage des aides sociales s'est développé en France de manière systématique assez récemment dans la législation française. Si la création du RMI en 1988 peut être considérée comme une façon de distribuer une allocation aux plus démunis, il faudra attendre une décennie pour que la France systématise le ciblage des aides plutôt que la politique visant à couvrir des risques à vocation universaliste, dans la lignée des protections offertes par le système de sécurité sociale datant de l'après-guerre.

Trois aides ciblées en particulier verront leur versement confié aux collectivités territoriales dans le cadre de la décentralisation par des majorités parlementaires différentes. L'allocation personnalisée d'autonomie, votée en 2001 ciblant les personnes âgées dépendantes, la prestation de compensation du handicap votée en 2005 et enfin le RMI puis son remplaçant, le revenu de solidarités actives mis en place en 2008 témoignent de ce changement affiché du traitement des politiques sociales françaises de l'universalisme vers le ciblage.

Parallèlement, des instruments visant à verser ces aides ciblées vont être créés par le législateur et vont se diffuser au sein des collectivités: les chèques de type Chèque emploi service universel ou Chèque d'accompagnement spécialisés.

Si l'émergence du "care " dans le domaine de la politique sociale (Laugier, 2011) permet de mieux percevoir les changements dans les modes d'intervention des pouvoir publics et l'émergence des services à la personne dans le champ de l'action sociale française, l'hypothèse que nous formulons est que cette notion ne peut expliquer à elle seule l'arrivée de systèmes de versement des aides ciblées par chèques au sein des collectivités locales françaises.

La notion de chèque, ou de "voucher " a en effet une filiation libérale et anglo-saxonne dans la mesure où l'utilisation du "voucher" a été conçue, dans sa version américaine, pour laisser sa liberté de choix au bénéficiaire de l'aide en particulier dans le domaine scolaire. Le pionnier de ces travaux sur le "school voucher " n'est autre que le monétariste Milton Friedman (Friedman, 1962) qui a d'ailleurs pu expérimenter cet instrument sous le régime de Pinochet au Chili (Garate, 2010, Hsieh, Urquiola, 2006).

Le système de chèque suit ainsi une des tendances du "nouveau management public ". Si l'on reprend certains des principes définis par Osborne et Gaebler (Osborne, Gaebler, 1992) pour "réinventer le gouvernement", il apparait en effet évident que le chèque est parfaitement compatible avec cette tendance qui a révolutionné la gestion publique (Christensen, Laegreid, 2001).

Ainsi, on retrouve dans le chèque l'idée que le gouvernement doit diriger plutôt que contraindre, introduire de la compétition dans les services délivrés au public, rencontrer les besoins des usagers, pas ceux de la bureaucratie (Hood, 1991). 
Toutefois, si l'idée du voucher a une filiation bien précise et fait l'objet d'une littérature très fournie, il apparait que le chèque tel qu'utilisé dans les collectivités françaises ne correspond pas tout à fait,chez les acteurs l'ayant mis en place, à la définition anglo-saxonne que l'on retrouve notamment chez Eugene Steuerle (Steuerle, 2000).

Afin de comprendre la façon dont le chèque a pu être accepté politiquement en France nous avons mené une étude doctorale sur plusieurs dispositifs de ciblage des aides individuelles utilisés dans les collectivités territoriales françaises afin de mieux définir le discours des acteurs autour de ces instruments. Cette étude a consisté en des recherches sur sources internes à plusieurs collectivités françaises (Conseils Régionaux Rhône-Alpes et Provence Alpes Côte d'Azur, Conseils généraux de Saône-et-Loire, de la Drôme et de I'Yonne), en une recherche documentaire principalement anglosaxonne et auprès des institutions financières internationales (Banque Mondiale, FMI) et de 45 entretiens semi-directifs avec les acteurs des collectivités ayant servi de terrain de recherche.

Les travaux menés au cours de cette thèse nous permettent de scinder cette communication en deux parties. Tout d'abord, nous définirons les caractéristiques du ciblage auregard notamment des travaux sur l'Etat Providence, puis nous nous intéresserons au chèque et à sa perception par les acteurs français, notamment suite à l' analyse des verbatimsen ayant assuré la mise en place et la gestion.

Nous constaterons ainsi au cours de cette étude que l'assourdissant silence ayant accompagné la mise en place massive des chèques comme instrument de ciblage des aides individuelles des collectivités françaises s'explique selon au moins deux facteurs que sont la technicisation du discours et la transformation de ces dispositifs en instruments de communication politique.

\section{Un instrument du ciblage}

Le chèque utilisé à des fins sociales ou plus généralement de remplacement d'une aide individuelle dans les collectivités françaises est un outil qui vise d'abord à cibler une population particulière avant de lui laisser un éventuel choix quant à son utilisation ou au contraire de restreindre ce choix (proscription). Nous pouvons donc émettre l'hypothèse que l'avènement du "care " dans les politiques sociales va permettre d'introduire le ciblage, notamment au regard de la notion d'Etat providence et des catégorisations dont cette notion a fait l'objet.

Les chercheurs(Esping-Andersen, 1990) s'étant penchés sur cette notion ont distingué plusieurs types de " welfare state " en étudiant en particulier la façon dont l'Etat intervenait en matière d'aide et d'assistance sociale. On a distingué les interventions ciblées appelées " targeting " visant certaines catégories de la population et les interventions " universelles » qui touchent la population toute entière.

Cette notion de ciblage en tant qu'outil voire en tant qu'instrument de politiques publiques apparait très liée au mode d'intervention par chèque des collectivités françaises et rejoint parfaitement les normes du Nouveau Management Public.

Cette catégorisation de la protection sociale dans l'Etat providence d'Esping-Andersen n'a pas énormément évolué, l'auteur s'inspirant de Richard Titmuss et de ces travaux datant des années 1950 (Titmuss, 1958). Le ciblage serait donc l'apanage des sociétés libérales et l'universalisme des sociétés « social-démocrate » (Esping-Andersen, 1999) 
Cette typologie est toujours utilisée comme référence dans la littérature. Kees van Kersberger et Philip Manow(Van Kersberger, Manow, 2008), ont redéfini la notion de " targeting " : Il s'agit pour eux d'un mode de versement des aides à la population visant à la fois à cibler un groupe social en particulier et à rendre l'aide la plus efficace possible.

Le ciblage d'une aide individuelle apparaît donc comme un outil de régulation sociale important et comme un critère déterminant de définition du type de société que l'on étudie.

On trouve cette logique de ciblage très souvent dans la littérature et particulièrement sur des sujets concernant le traitement de la pauvreté dans les pays en difficulté. Nous retiendrons la définition du " targeting » exposée par Laure Pasquier-Doumer, Emmanuelle Lavallée, Anne Olivier, Anne-Sophie Robilliard dans la Revue d'économie du développement en 2009 (Pasquier-Doumer et al, 2009).

Etablissant un bilan des expériences de la lutte contre la pauvreté dans certains pays, elles définissent cette notion comme "la concentration des ressources des programmes de lutte contre la pauvreté sur les populations les plus pauvres ou les plus vulnérables. "

Les auteurs voient le début de ce recours aux politiques ciblées dans les pays en voie de développement dans les années 1980. II s'agissait au départ de compenser les effets des plans d'ajustements structurels suite notamment à la "crise de la dette " et aux interventions des institutions financières internationales, tout en maintenant la rigueur budgétaire imposée à l'époque.

Elles estiment que, dans les années 1990, un changement de paradigme intervient dans l'aide au pays pauvres : la réduction de la pauvreté devient alors un objectif prioritaire pour la communauté internationale. C'est la transformation de la définition du ciblage, ou plutôt de son utilisation qui accompagne ce changement. Le «targeting » change ainsi de statut.

Défini au départ comme un "outil d'accompagnement", il devient un "instrument pour la recherche d'une plus grande efficacité de la lutte contre la pauvreté ".

Ce changement de statut peut d'ailleurs être à rapprocher de la notion d'instrument d'action publique telle que définie par Pierre Lascoumes et Patrick Le Galès. Si I'on relit la définition couramment admise, un instrument d'action publique est " un dispositif technique à vocation générique porteur d'une conception concrète du rapport politique/société et soutenu par une conception de la régulation » (Lascoumes, Le Galès, 2004).

Cette notion d'instrument, si elle peut éventuellement s'appliquer à la notion de ciblage telle que définie dans une très riche littérature et dans de nombreuses études menées par les organisations internationales dont la Banque Mondiale et le Fonds Monétaire International sont les fers de lance, est loin d'être évidente lorsque nous l'appliquons à l'objet de l'étude.

Dans le cas de la définition du ciblage, les institutions utilisatrices de ce mode d'intervention partent du principe, a priori séduisant, que la réduction de la pauvreté sera plus efficace si les ressources sont concentrées sur les populations les plus pauvres plutôt que si elles sont distribuées à toute la population par le biais d'une politique universaliste.

Le ciblage est donc conçu comme une politique globale de lutte contre la pauvreté, dont l'usage apparait généralisé. Les principales études proviennent de chercheurs évoluant dans les institutions internationales. Les types d'interventions du ciblage ont été notamment étudiés par David Coady, Margaret Grosh et John Hoddinot (Coady, Grosh, Hoddinot, 2004) qui en distinguent cinq : les transferts monétaires directs, les transferts en nature, les subventions à la consommation, les travaux publics, et les fonds sociaux. 
Parmi ces modes d'interventions, on en retrouve quatre qui peuvent être conditionnés au respect de certaines obligations par les bénéficiaires. On se retrouve alors en présence d'une forme de ciblage particulière: le transfert monétaire conditionnel ou "conditional cash transfer" (Kervyn de Lettenhove, 2012).

Seule une catégorie correspond à la définition du "chèque " ou "voucher ", il s'agit du transfert en nature qui ne semble pas répondre à la même préoccupation que le "voucher " anglo-saxon, même si formellement, il s'agit de la même chose (un titre de paiement d'un bien ou d'un service).

Il s'agit ici davantage de contrôler l'utilisation des fonds publics (en donnant des bons alimentaires plutôt que de l'argent par exemple) plutôt que de laisser la liberté de choix au bénéficiaire de l'aide. On retrouve donc tout de même un des objectifs pouvant justifier une politique de "vouchers » tels que définis notamment par Eugene Steuerle (Steuerle, 2002).

Pour les promoteurs du ciblage, non seulement, la population doit être ciblée, mais l'aide doit être octroyée en contrepartie d'une action particulière, d'un changement de comportement de la part du bénéficiaire (scolariser ses enfants, se soigner, équiper son logement...) Cette idée de transfert monétaire ciblé dépasse d'ailleurs les frontières du domaine de l'aide aux pays en développement.

C'est cette notion plutôt que celle de "voucher " qui s'applique donc davantage à la notion de " chèque emploi service " telle qu'on la trouve en France et qui remplace dans un quart des Conseils Généraux une ou plusieurs aides sociales obligatoires.

Les dispositifs ciblés ont d'abord été testés en Amérique du Sud, pour des raisons géographiques, mais surtout historiques et institutionnelles. En Amérique du Sud comme dans d'autres anciennes colonies, les "institutions du pillage » dites "plunder institutions " mises en place par les pouvoirs coloniaux ont persisté bien après l'indépendance, aggravant ainsi les inégalités et pénalisant durablement la croissance de ces pays (Acemoglu, Johnson, Robinson, 2001).

C'est cette inégalité originelle, à laquelle vont s'ajouter les conséquences dramatiques de la " crise de la dette " et son apogée avec la faillite de l'Argentine en 2001-2002, qui semble avoir provoqué une explosion des inégalités.

La pauvreté atteindra alors un niveau difficilement soutenable et rendra obsolètes les moyens " classiques " utilisés jusqu'alors, qui mêlaient un clientélisme politique et un subventionnement important (et assez contre-productif) des produits de première nécessité, notamment au Mexique (Lautier, 1998).

Les politiques ciblées en Amérique du Sud sont supposées lutter contre le dévoiement des anciennes politiques sociales : d'abord, le subventionnement des biens de première nécessité profiterait plus à ceux qui n'en ont pas besoin, et qui consomment plus que les pauvres, ensuite, il convient de lutter contre les abus des politiques trop universelles dont beaucoup de bénéficiaires abuseraient. Le ciblage est donc, d'après Bruno Lautier, clairement un outil de sélection de ceux qu'il faut aider, en opposition à ceux qu'il ne faut pas aider (Lautier, 2010).

Promues à la fois par les institutions internationales et par les gouvernements nationaux, les politiques sociales ciblées sont souvent très hétérogènes et se caractérisent non pas par la nature de l'intervention ou de la population ciblée, mais par la technique utilisée.

Bruno Lautier recense plus d'une centaine de ces politiques dans toute l'Amérique du Sud entre 1990 et 2010. Les nombreuses monographies visant souvent à évaluer les politiques ciblées dans cette région montrent en effet que ces modalités de versement des aides se sont multipliées durant cette période. Parmi les "grands" pays américains, on retiendra la BolsaFamilia Brésilienne, le 
programme Chile solidaria au Chili ou encore Oportunidades au Mexique qui ont survécu aux alternances politiques et sont des piliers des politiques d'insertion de ces Etats.

Les évaluations ponctuelles que l'on trouve sont d'ailleurs assez optimistes. Dès 1994, Margaret Grosh (Grosh, 1994), qui passe en revue une trentaine de dispositifs en Amérique Latine, tire deux conclusions de son étude :

- Les programmes ciblés ont une incidence beaucoup plus importante sur la réduction des inégalités que les politiques de subventionnement des produits alimentaires, et même que les politiques générales de santé et de scolarisation des enfants.

- Les coûts administratifs de ces dispositifs n'ont pas besoin d'être excessivement élevés.

Ces conclusions, assez encourageantes, seront reprises et partagées dans une autre étude comparative de trois grands systèmes de ciblage réalisée par Natalia Caldés, David Coady et John Maluccio publiée 10 ans plus tard (Caldès, Coady, Maluccio, 2004).

Cet article commence toutefois à souligner des difficultés et des échecs relatifs rencontrés par ces politiques dont les écueils apparaissent de façon importante.

\subsection{Identifier les populations : les erreurs d'inclusion et d'exclusion}

Les aides ciblées ayant fait l'objet d'évaluations ont été mises en place en majorité dans des pays en développement et ont ciblé les populations les plus pauvres. Le principe, a priori de bon sens, étant que si les inégalités sont importantes, il conviendra de concentrer les efforts d'une politique publique sur la frange la plus en difficulté pour que les écarts tendent à se resserrer.

Dans la pratique, les études de terrain ont mis en évidence plusieurs écueils et difficultés. La principale difficulté réside dans l'identification du "pauvre ». Dans une région où l'information est imparfaite, un programme de réduction de la pauvreté ne peut se permettre de commettre des erreurs d'inclusion (en faisant bénéficier de son aide des personnes qui ne sont pas dans la cible) ou d'exclusion (en oubliant des personnes qui sont dans la cible car elles ne seraient pas répertoriées).

Deux méthodes de sélection des populations ont été testées : I'auto-sélection qui vise en la mise en œuvre d'incitations qui encouragent la participation des populations les plus pauvres tout en décourageant les moins pauvres et la collecte d'informations sur les conditions de vie des ménages qui permet de séparer les populations ciblées des populations non ciblées. Evidemment, la collecte d'information connaît des résultats qui varient en fonction de la solidité institutionnelle du pays où le programme de ciblage est testé et de son niveau de corruption (Smith, Subbarao, 2003).

Par ailleurs, cette méthode, la moins coûteuse a priori, tendrait, dans les pays en voie de développement, à déléguer ce pouvoir à des représentants pas forcément légitimes (au sein de clan ou de tribus pour reprendre l'exemple de l'Amérique du Sud où les Indiens sont la plupart du temps au cœur des populations à cibler en priorité) et donc à perpétuer un certain clientélisme qui non seulement ne permet pas une nouvelle fois d'atteindre la cible visée mais ne fait que renforcer les chefferies locales (Lautier, 2010).

En toute logique, ce type de biais devrait toutefois être assez limité dans un pays ou une région où les données sont simples et fiables.

D'après les analystes de ce type de programmes, les erreurs d'inclusion conduisent à un gaspillage des ressources et augmentent le coût du programme sans améliorer son efficacité. On parle de 
diminution de l'efficacité "verticale » du programme dans le langage de la Banque Mondiale (Bibi, Duclos, 2007).

Au contraire, les erreurs d'exclusion diminuent le coût du programme au détriment de son efficacité, elles créent des inégalités "horizontales " (entre personnes ayant le même niveau de revenus) et peuvent conduire à de l'instabilité sociale et donc à l'inverse de ce que le programme d'aide recherche.

Bruno Lautier voit dans ces difficultés de repérage des cibles à privilégier non seulement un problème technique, mais surtout un problème d'ordre idéologique :

"Les cibles désignées sont prioritairement les plus "visibles ", celles qui ont le plus de poids politique, et celles qui correspondent aux a priori de ceux qui sont chargés de mettre en œuvre ces politiques. Cela provoque des effets de mode, qui poussent à mettre en avant, à certains moments, certaines catégories de population (les femmes, les enfants, les "indigènes "...), et à en oublier, le plus souvent, d'autres (les travailleurs âgés en milieu urbain, ou certaines catégories de workingpoor, en particulier les femmes chefs de famille). " (Lautier, 2010).

\subsection{Une nécessité d'un soutien politique de la population}

L'autre inconvénient du ciblage relevé par les experts des organisations internationales est d'ordre politique. Certains analystes de la Banque Mondiale reprenant les notions de " meritgoods " (Becker, 1974) ou de "public value " (Moore, 1995) considèrent qu'en excluant des bénéficiaires une partie de la population, la politique ciblée ne bénéficiera pas du soutien de la population la plus active politiquement et notamment la classe moyenne qui, dans les pays en développement, forme souvent la frange clé de l'électorat au sein de la société et est donc dotée d'un poids électoral important (Pasquier-Doumer et al, 2009). On touche ici à l'un des fondements du nouveau management public qui est l'importance du lien entre le client / contribuable et le gouvernement.

Cette puissance de l'électorat constitué par ces classes moyennes explique certains échecs des politiques ciblées de lutte contre la pauvreté. Ainsi, Jonah Gelbach et LantPritchett estiment que le modèle économique, utilisé par la Banque Mondiale notamment, considérant que les subsides doivent être ciblés vers ceux qui en ont le plus besoin, sera au final contre-productif car sous la pression d'enjeux électoralistes (Gelbach, Pritchett, 2002).

Le politique devra au final faire baisser le financement de son programme ciblé, le rendant moins efficace qu'un programme à vocation universelle.

Ce raisonnement est également présent chez des chercheurs de I'OCDE. Ainsi, Emma Cain considère qu'en se concentrant sur les personnes les plus pauvres, les programmes ciblés ont un faible soutien politique, apparaissant alors comme étant une aide réservée à une minorité plus que comme un programme profitant à tous au contraire des dispositifs universels qui apparaissent comme des " acquis sociaux » qui résisteront mieux à des changements politiques ou à des chocs économiques (Cain, 2009).

Cette analyse est reprise dans une note d'orientation de l'OCDE publiée en 2008 qui recommande une limitation des programmes ciblés à certains cas très particuliers (OCDE 2008). L'OCDE, dans cette étude, considère que les programmes ciblés n'ont qu'un avantage évident : leur coût administratif, bien inférieur à celui d'un programme universaliste.

De même, dans une étude comparative sur les erreurs d'inclusion et d'exclusion de programmes ciblés dans 9 pays, Giovanni Cornia et Frances Stewart arrivent à la conclusion que le fait de 
supprimer un programme universel pour cibler davantage une population en particulier est un facteur qui, au final, peut amener les prestations réelles moyennes versées aux individus à diminuer pour un coût similaire pour les finances publiques (Cornia, Stewart, 1995).

Le faible soutien politique peut donc transformer les « programmes pour les pauvres en pauvres programmes" pour reprendre l'expression de ThedaSkopcol qui est l'une des partisanes d'une politique mixte de « ciblage dans l'universel » (Skopcol, 1991).

En s'appuyant sur le fait que les politiques d'aides aux noirs américains à vocation universelle (qui visent tous les noirs et pas seulement les pauvres) bénéficient davantage aux plus nécessiteux et sont mieux acceptées par la population, ThedaSkopcol observe que le ciblage ne peut fonctionner que s'il ne défavorise pas la classe moyenne.

A l'inverse, dans certains cas, la population peut soutenir la mise en œuvre de politiques ciblées plutôt que de politiques universelles. II faut pour cela que la population perçoive qu'il y a un moyen d'atteindre efficacement les pauvres en économisant des ressources afin de financer d'autres biens publics. Laura Tuck et Kathy Lindert ont notamment étudié comment une campagne de sensibilisation avait contribué, en 1989 en Tunisie, à faire accepter par la population la transformation d'un programme universel d'aide alimentaire en un programme ciblé (Tuck, Lindert, 1996).

La communication autour de ce programme a consisté en une démonstration selon laquelle la mesure d'aide universelle était inefficace. Une enquête menée avant la réforme avait révélé que l'opinion publique estimait que le programme précédent était trop coûteux et qu'il fallait le transformer.

L'hypothèse selon laquelle la conditionnalité de l'aide et le fait de ne pas donner de liquidité directement permet de mieux faire accepter le dispositif a d'ailleurs été étudiée par Dominique Van de Walle qui souligne qu'il est possible de rendre acceptables par la population des programmes ciblés en modifiant les modalités de versement de l'aide, introduisant la notion de "transferts monétaires conditionnels » ou " Conditional cash transfers " (Van de Walle, 1998).

Il estime ainsi que l'opinion publique est généralement moins hostile au ciblage des transferts en nature ou de programmes lorsque ces derniers impliquent des contreparties (notamment en termes de travaux d'intérêt généraux ou de modification de comportement) qu'au ciblage de transferts monétaires inconditionnels.

\subsection{Ciblage et conditionnalité des aides individuelles}

Les politiques ciblées semblent donc, depuis les années 1980, être les outils préférés des organismes financiers internationaux pour lutter contre la pauvreté. Selon eux, il vaudrait en effet mieux identifier, puis aider les pauvres, plutôt que de bâtir des programmes universalistes comme des systèmes de couverture sociale universelle, d'assurance maladie ou d'assurance chômage.

Le ciblage reste l'instrument favori des grandes institutions internationales et la littérature généraliste, mais aussi monographique sur le sujet est principalement issue de cénacles tels que la Banque Mondiale, le Fonds Monétaire International mais aussi l'ONU, à travers la FAO ou I'Unicef.

Bien que les publications actuelles sur le ciblage aient tendance à remettre en cause l'efficacité de ce modèle, il semble que celui-ci soit porté par un courant libéral dont il apparaît qu'il ne permet pas réellement de proposer des choix alternatifs. 
Ce panorama rapide du ciblage des aides sociales dans les politiques de lutte contre la pauvreté, nous permet également d'opérer une distinction entre le ciblage de la population au sens "targeting " et le fléchage de l'aide qui cherche à ce que les fonds alloués soient utilisés le plus efficacement possible par la population ciblée. C'est en mêlant ces deux objectifs que le chèque ou "voucher " a pu devenir un instrumentprivilégié des politiques culturelles et sociales au sein des collectivités locales françaises.

\section{Le chèque comme instrument dépolitisé du «targeting »}

L'hypothèse que nous avons développée dans la recherche est que les chèques, présentés par ses promoteurs en France comme de simples outils de communication à destination des collectivités territoriales (Lacheret, 2014) constituent de véritables instruments (Lascoumes, Le Galès, 2004) ou plus précisément d'outils (Salamon, 2001) qui permettent de développer le ciblage des aides individuelles.

L'évolution de la politique sociale passe en effet par plusieurs innovations importantes qui ont contribué à modifier les modes d'actions des acteurs publics se chargeant de distribuer les aides. L'intégration de la notion de "social care " dans l'arsenal des politiques sociales est la première d'entre elles.

Cette arrivée du "care " est d'abord liée à des facteurs tels que la montée en puissance de la problématique de la dépendance des personnes âgées qui a notamment vu exploser les montants des budgets, mais aussi le nombre de modes d'intervention et, bien sûr, la littérature, sur les soins aux personnes, qu'elles soient âgées, dépendantes, en situation de handicap voire en recherche d'emploi.

Le deuxième élément qui fonde notre hypothèse est que les politiques sociales " universalistes » de type assurantielles n'incarnent plus la notion d' "innovation ». Les nouveautés de l'aide sociale, en France, mais aussi, nous l'avons vu à travers une littérature très riche, partout dans le monde, consistent en la multiplication de dispositifs ciblés

Ainsi, dans l'introduction générale d'une étude sur l'innovation sociale publiée en 2009, il est d'emblée précisé que les politiques sociales universalistes françaises ont atteint les objectifs qu'elles s'étaient assignées, mais que les mutations sociales rendent nécessaire le recours à l'innovation. La première série de questions posées par les auteurs illustre assez bien le fait que, pour eux, l'universalisme a fait son temps (Damon, 2009).

Cette logique d'un ciblage plus important des aides qui nécessiterait la mise en place d'outils novateurs se retrouve également dans les productions des agences de l'Etat en charge de la réflexion et de l'organisation des nouvelles politiques sociales françaises. Ainsi, l'Agence Nouvelle des Solidarités Actives, associée pour l'occasion à un émetteur de Chèques emplois services universels s'appuie, dans l'introduction de son étude intitulée "Aides sociales, enjeux et pratiques locales ", sur l'augmentation du nombre de travailleurs pauvres pour justifier la création d'un dispositif tel que le Revenu de Solidarités Actives.

\subsection{Un chèque encore trop assimilé au « libre-choix "}

L'approche anglo-saxonne du chèque destiné à obtenir un service social, reprise par les organisations internationales, demeure toutefois beaucoup trop axée sur l'idée de laisser une liberté de choix au 
bénéficiaire, souvent traduit par le terme " customer » dans les articles traitant du sujet ce qui relie une nouvelle fois cet outil au Nouveau Management Public.

Les études d'impact et d'efficacité des chèques services à but sociaux outre-Atlantique ont souvent, à juste titre, soulevé le problème des fournisseurs de services qui parfois pouvaient être sujets à des dérives sectaires ou à du prosélytisme religieux à cause notamment d'une absence de contrôle corolairede cette idéologie du "libre-choix ». C'est le cas notamment d'une étude sur deux dispositifs importants: le "child care developmentfund" et le "temporary assistance for needyfamilies » (Burwick, Kirby, 2007).

Ce lien entre chèque-service et "libre-choix » est également repris par une étude de l'OCDE dirigée par Jens Lundsgaard. Cherchant à contourner les problèmes d'asymétrie de l'information entre les pouvoirs publics et les prestataires financés par des fonds publics, Jens Lundsgaard propose plusieurs solutions parmi lesquelles la mise en place d'un système de modulation des financements en fonction des performances des prestataires, la réalisation d'évaluations comparatives, le recours à la sous-traitance ou une formule de chèque-service. Ce système de chèque, "tout en en laissant à l'usager la possibilité de choisir parmi différents prestataires proposés, n'interrompt pas la manne des financements publics » (Lundsgaard, 2002)

Plus loin, il définit le principal avantage de ce "chèque service " comme étant une façon originale d'ouvrir un service à la concurrence "qui consiste à laisser à chaque usager la possibilité de choisir son prestataire parmi ceux qui lui sont proposés. " Lundsgaard observe toutefois plusieurs écueils possibles, et tout particulièrement le respect de la libre concurrence.

En effet, tout en se montrant confiant sur la capacité du bénéficiaire à effectuer un choix parfaitement libre, l'auteur estime que certaines personnes, et notamment les personnes âgées dépendantes, n'ont pas la capacité de choisir librement et peuvent "éprouver quelque difficulté à comparer les services proposés par divers établissements de long séjour et ne pas être en mesure de faire le choix le plus conforme à [leurs] intérêts. ". Cet écueil peut être surmonté, notamment en mettant en place des procédures d'agrément des prestataires qui garantiraient une qualité de service minimale aux usagers.

Il observe également le problème des "monopoles locaux ", particulièrement importants dans les zones rurales ${ }^{2}$.

Par ailleurs, il identifie une autre difficulté dans certaines pratiques que peuvent avoir les prestataires privés, notamment en favorisant les bénéficiaires "faciles " et en discriminant les personnes âgées très dépendantes, nécessitant des soins trop lourds en les orientant, par exemple, vers des établissements spécialisés. II suggère entre autres aux pouvoirs publics de moduler le financement en fonction des caractéristiques des usagers, de façon à ce que les soins apportés au public le plus « difficile » soient plus rémunérateurs.

Enfin, il soulève un phénomène fréquemment observé : les formules de chèques-services peuvent entraîner au final une hausse globale de la demande, des prix (par un effet d'aubaine), et au final du coût pour le financeur public.

II propose, là aussi, « de prévoir, en amont de la prise de contact entre l'usager et le prestataire, une évaluation des besoins individuels par un organisme indépendant afin de déterminer si les conditions $d^{\prime}$ attribution des prestations sont remplies. La solution qui consiste à fixer autoritairement, comme dans le système de chèques service, le montant des subventions publiques individuelles (en fonction de la situation de chaque usager) tout en laissant aux usagers la possibilité de les compléter par leurs

\footnotetext{
${ }^{2}$ En France, les ADMR sont souvent citées lors des entretiens avec les acteurs comme étant en situation de monopole, nous avons pu le constater durant l'enquête de terrain.
} 
propres versements peut permettre de résorber la demande excédentaire dans la mesure où dans ce système, ce sont les usagers qui supportent l'intégralité des coûts marginaux. "

Cette solution ne serait valable selon l'auteur que dans certains types de prestations et tout particulièrement lorsqu'il s'agit de soins aux personnes âgées dépendantes mais serait plus complexe lorsqu'il existe un " monopole local » II suggère dès lors soit de laisser au bénéficiaire le soin de payer le coût marginal occasionné ou alors de fixer les prix de façon autoritaire afin d'éviter que les monopoles ne s'en chargent.

Afin d'éviter que les prestataires n'influent trop largement sur le niveau de la demande, en augmentant les prix par exemple, l'autorité publique pourrait limiter le nombre d'agréments délivrés ou passer par un système de numerus clausus. La plupart de ces recommandations, formulées dans cette étude de I'OCDE publiée en 2002 ont été reprises lors de la loi instaurant l'Allocation Personnalisée d'Autonomie, puis dans la loi de cohésion sociale permettant aux collectivités de verser le montant de certaines prestations sociales en Chèque.

\subsection{Image et représentation du chèque en France}

Le chèque en tant qu'outil a été introduit massivement en France, que cela soit en instrument de remplacement d'aides légales existantes (APA, PCH) où désormais 23 Conseils Généraux ont remplacé partiellement ou totalement le versement en numéraire par des CESU ou des Chèques d’Accompagnement Personnalisé ou pour créer des aides ciblées facultatives.

Pour ce dernier cas, les exemples sont très nombreux (Lacheret, 2014, Rouet, 2009). 21 régions métropolitaines sur 22 et plus de la moitié des Conseils généraux ont mis en place ce type d'aide ciblant une catégorie de la population. On retrouve majoritairement des dispositifs d'aide à l'acquisition de livres scolaires ciblant les lycéens, souvent doublés d'aides à l'accès à la culture ou aux sports. Une dizaine de Régions ont également adopté des chèques "énergies renouvelables " ciblant les ménages désireux d'équiper leurs logements en chauffe-eau solaire, en chaudière à bois ou autres.

Si les chèques ont souvent été porteurs d'un sens politique très libéral au regard des idées de leur théoricien (Friedman, 1962), les efforts des politiques ont visé à le dépolitiser, souvent par le biais de mécanismes d'évaluation (Lacheret, 2013, Mac Ewan, 2004) afin de les rendre plus acceptables politiquement. En France, on a assisté à un processus un peu différent visant à camoufler le sens politique originel du chèque.

La façon dont ces instruments ont été présentés, les discours ayant permis leur introduction et les diverses médiations dont ils ont fait l'objet ont permis, dans un silence idéologique assourdissant, de multiplier les dispositifs de "targeting " considérés comme néolibéraux ailleurs dans le monde en camouflant un sens politique qui aurait sans doute rendu beaucoup plus complexe leur adoption par les collectivités locales françaises.

Toutefois, aucun indice apparent ne pouvait laisser entendre qu'un outil qualifié de néolibéral aux Etats-Unis et autour duquel les débats furent passionnés tant au sein de la communauté scientifique qu'au niveau des débats politiques fédéraux pouvait être comparable à un dispositif utilisé par les Conseils Régionaux et Généraux pour verser des aides culturelles ou sociales.

Les écrits de Friedman datent des années 60 et semblent en effet ne pas avoir influencé directement les élus régionaux et départementaux français au point de les pousser à reproduire son modèle. De surcroît, dans les années 2000, la plupart des Régions et Départements sont dirigés par des exécutifs de gauche dont les discours sont éloignés de la rhétorique du père du monétarisme. 
Et pourtant le chèque va envahir les collectivités françaises en quelques années, devenant un outil idéal du ciblage des aides sociales, mais également un outil de communication, permettant de matérialiser l'aide publique.

\subsubsection{Enquête de terrain et analyse des verbatims}

L'enquête de terrain, qui a mobilisé 45 entretiens répartis entre différents acteurs des terrains choisis (Conseil régional Rhône-Alpes, Conseils généraux de la Drôme et de la Saône et Loire qui sont trois des collectivités pionnières dans l'usage du chèque) avait pour objectif de vérifier une hypothèse centrale selon laquelle le voucher anglo-saxon et les chèques français étaient un seul et même objet. Si physiquement et par l'usage qui en est fait, il est simple de répondre à cette question, le plus important était de pouvoir prouver que les arguments développés pour décrire le chèque utilisés par les chercheurs anglo-saxons correspondaient à ceux employés par les acteurs interrogés.

L'enquête a donc en effet consisté en une analyse des verbatims des acteurs, en recoupant les arguments qu'ils développaient et en les croisant avec ceux définis par les théoriciens et analystes anglo-saxons du " voucher " donnant à cet outil en apparence neutre une connotation néolibérale que les acteurs ne revendiquent pas ou très peu.

Questionner la représentation d'un objet et le sens que l'interlocuteur lui donne relève de l'approche phénoménologique. Alex Mucchielli (Mucchielli, 1997), dans le dictionnaire des méthodes qualitatives en sciences humaines et sociales indique que le but de cette approche est de « recueillir de multiples descriptions de mêmes objets ou phénomènes provenant de sujets différents pour essayer de trouver ce qu'il y a de commun à ces différentes approches. "

II nous aura donc fallu emprunter à cette approche pour permettre à chaque acteur de donner une représentation d'un même objet : le chèque. Le fait de choisir et de sélectionner un échantillon sera, pour Mucchielli : " une opération mentale qui suppute les différences et les analogies et qui conclut à la validité du recueil, car la situation sujet-objet concerne la recherche. Dans cette opération, le processus intellectuel de comparaison est à l'œuvre ". Le choix de la qualité de la personne interrogée nécessite donc une opération intellectuelle, une comparaison de la part du chercheur.

Le choix a été fait d'interroger plusieurs catégories d'acteurs en bâtissant un questionnaire semidirectif dont les questions étaient très proches (à quelques questions et relances près ayant trait au positionnement de l'acteur dans l'organigramme de la collectivité). L'essentiel étant de rassembler tous les acteurs du chèque au sein de la collectivité quel que soit l'échelon auquel ils évoluaient.

Ainsi, 14 élus, 9 cadres dirigeants (Directeurs généraux), 16 cadres intermédiaires (chefs de services, chargés de mission) et 6 personnalités extérieures (salariés d'entreprises commercialisant les chèques) ont été interrogés sur leur représentation du chèque en passant également par le sens politique qu'il donnait à cet outil.

Les verbatims ont ensuite été croisés avec les 6 critères revenant le plus souvent chez les chercheurs et synthétisés par Eugene Steuerle (Steuerle, 2002) :

- La notion de libre-choix ("choix et efficacité " pour Eugene Steuerle) que donnerait à l'usager la possession d'un chèque, est présente tout particulièrement dans les discours et arguments développés à tous les niveaux hiérarchiques de la chaîne de décision et de gestion des chèques destinés au jeune public (Steuerle, 2000). 
On peut illustrer ce discours sur le libre-choix avec Amaury Nardone (10 février 2010), ancien Président de la Commission culture du Conseil régional Rhône-Alpes : "le seul moyen de faire une politique culturelle dans laquelle le politique n'est pas un censeur, c'est de donner le choix au citoyen. C'est de donner le choix au citoyen de subventionner - pas d'acheter hein! - de subventionner, l'institution culturelle, le spectacle, le musée, le bouquin même, puisque le chèque culture va jusqu'au livre, qu'il a envie de subventionner. "

- On trouve également chez les acteurs la notion de chèque comme moyen de faire accepter une aide par la population (" choix et équité »), que Steuerle positionne comme le revers de la médaille du libre-choix. L'équité permet de créer de la «public value ", faisant accepter le chèque par le client/contribuable. Cette notion est présente de façon assez constante chez les acteurs des chèques à vocation sociale, mais aussi de façon assez systématique à tous les niveaux de décision.

Ainsi le Directeur Général des Services du Conseil général de la Drôme (11 juillet 2011) indique que "Le CESU a été implanté pour éviter tout gaspillage [...] les gens sont pour qu'on contrôle, même avec le RSA»

- Dans la littérature, l'argument le plus libéral au sens économique du terme justifiant I'utilisation du chèque reste toutefois celui selon lequel l'utilisation du chèque permet d'accroitre la compétition entre les acteurs et donc, par les bienfaits supposés de la concurrence, de créer un marché plus compétitif et plus attractif pour le bénéficiaire/consommateur. On le trouve à la fois dans les propos des acteurs du chèque culturel et du chèque de type "énergies renouvelables». Pour ce dernier, l'argument du marché est plutôt développé de façon négative. Le chèque a en effet permis de structurer le marché, mais l'aide offerte a faussé ce dernier. Les installateurs ont plutôt augmenté leurs tarifs en se multipliant (ce qui est l'exact inverse de la théorie économique voulant que dans un libre marché, les prix baissent avec l'augmentation du nombre d'acteurs), créant une confusion et parfois un rejet dans la représentation des acteurs.

Pour illustrer ce type de réponses, très fréquentes, on citera notamment un extrait de l'entretien avec le chef du service sport/jeunesse au Conseil général de la Drôme à propos du chéquier destiné aux collégiens et de la concurrence entre partenaires de l'opération : "On s'est rendu compte que dans le secteur associatif, ceux qui ont vraiment joué le jeu ont eu beaucoup de bénéficiaires »

- Le chèque peut également être présenté par les acteurs comme un outil servant à remplacer un autre programme, une autre politique publique (c'est également un argument important $d^{\prime}$ Eugene Steuerle). Le chèque a en effet été l'outil de mise en place ou de remplacement de plusieurs programmes et est bien perçu comme tel par les acteurs, notamment lorsqu'ils évoquent les chèques sociaux, mais aussi les chèques environnementaux et culturels.

On peut, parmi d'autres, reprendre les propos de la responsable du service énergie au Conseil Régional Rhône-Alpes (28 avril 2013) : " Auparavant, on avait un dispositif d'aide qui passait par la subvention, donc là ce sont nos dispositifs classiques d'interventions financières, c'est ce dispositif-là qui avait explosé qui était devenu difficile à maitriser, et donc au moment où on a décidé de passer en chèque, pour pouvoir avoir une adaptation du volant de ressources humaines affectée, dans la fonction publique, c'est compliqué, quand on change les effectifs, ça passe par des concours administratifs, pour recruter des fonctionnaires, c'est quand même très long, ou alors on est obligés 
de faire appel à des temps partiel ou des contractuels, et on est limité dans le temps, c'est compliqué, il faut justifier que le dispositif n'est pas pérenne donc il nous semblait que justement le chèque énergie permettait cette souplesse là en fonction des options politiques qui seraient votées ou pas, donc l'idée c'était bien à la fois de maitriser par l'études de nouveaux critères l'inflation du dispositif, donc de revenir à des choses qui budgétairement était plus maitrisables, et d'un autre côté, d'externaliser l'exécution de la mise en œuvre et donc ça c'est le passage au chèque énergie. "

- La limitation du choix du bénéficiaire - le chèque prescrit et proscrit en même temps (Bradford, Shapiro, 1999) est également un argument présent majoritairement chez les acteurs des chèques sociaux qui y voient un moyen efficace de limiter le recouvrement des "indus » lorsque des aides sont dépensées pour autre chose que leur destination initiale. Le chèque est ici représenté comme un outil de contrôle de la dépense sociale.

On peut retenir la façon dont Pierre Bucco (31 janvier 2013), ancien Directeur Général adjoint des Services au Conseil général de Saône et Loire, illustre cet argument en parlant du chèque départemental permettant de verser la Prestation de Compensation du Handicap : " I/ s'agissait de mettre en place un système qui nous permette très rapidement de contrôler que ça avait été fait, ça avait été utilisé, et si ça n'avait pas été utilisé, du coup, on diminuait d'autant les chèques à donner. Le système il est simple: "Madame, vous avez droit à tant d'heures, ça fait tant de chèques "- mettons que la valeur faciale est de 17 ou 18 euros par chèque... donc on vous envoie le chèque et les services, du coup c'était Chèque déjeuner, nous envoyaient très rapidement, dans les deux mois, il y avait toujours un petit décalage de deux mois, les personne qui avaient utilisé leurs chèques, et donc du coup, on savait que ces personnes-là il leur restait des chèques pour certains. Et donc le mois d'après, on passait la commande à Chèque déjeuner, en disant, pour Madame Machin, vous diminuez le chéquier de X chèques parce qu'on savait qu'elle avait gardé les autres chèques.»

- Enfin, l'argument financier selon lequel le versement d'une aide publique en chèque serait une source d'économies est également parmi les plus développés par les acteurs du chèque social, tout particulièrement par les élus et les cadres dirigeants des collectivités. On peut également identifier des bribes d'arguments de type économique chez les gestionnaires $\mathrm{d}^{\prime}$ autres dispositifs (chèques à destination des jeunes publics en particulier).

Boris Vallaud (3 mars 2013), Directeur Général des Services du Conseil général de Saône et Loire est très disert sur le sujet, tout comme la plupart de ses collègues : "La réflexion vient des services eux-mêmes, services $P A-P H$, qui savent qu'il y a un sujet, que devant l'explosion des dépenses sociales, il faut autant que faire se peut les contrôler, faire du contrôle de gestion, on sait la difficulté qu'il y a à récupérer les impayés, les indus, les choses comme ça, on sait que ça demande des moyens humains que l'on n'a pas et que l'effectivité de la dépense, ça peut représenter des sommes colossales, et on peut récupérer beaucoup plus d'argent à travers le chèque que avec des contrôles classiques. Et c'est indolore pour les personnes parce que finalement si elles n'ont pas utilisé le chèque, elles ne l'ont pas utilisé. "

L'hypothèse formulée était que si ces arguments se retrouvaient tous dans les discours des acteurs, il était vraisemblable que l'objet chèque était non seulement le même en terme d'usage, mais aussi en terme de définition politique. 
Après analyse des résultats, on peut donc effectuer une synthèse finale des arguments présents dans la littérature et évoqués par les acteurs du chèque au sein des collectivités locales en fonction des thématiques des chèques. II revient que les arguments financiers, de l'acceptabilité par la population (équité) et de limitation du choix du bénéficiaires reviennent plus souvent et sont plus détaillés dans les propos des acteurs évoquant un chèque de type "social", le libre-choix du public, le renforcement de la compétition et le remplacement d'un programme existant entre acteurs étant des arguments plus marqués dans les propos des acteurs évoquant des chèques de type " jeunesse " ou « énergies ».

On peut illustrer la récurrence des arguments qualifiant le chèque dans le discours des acteurs en fonction des thématiques des dispositifs étudiés à travers le tableau suivant :

\begin{tabular}{|l|l|l|l|}
\hline & Social & Jeunesse & Energies \\
\hline Libre-choix & & & \\
\hline Equité & & & \\
\hline $\begin{array}{l}\text { Compétition } \\
\text { entre acteurs }\end{array}$ & & & \\
\hline $\begin{array}{l}\text { Remplacement } \\
\text { d'un } \\
\text { programme } \\
\text { existant }\end{array}$ & & & \\
\hline $\begin{array}{l}\text { Limitation du } \\
\text { choix } \\
\text { bénéficiaire/ du } \\
\text { Contrôle de la } \\
\text { dépense } \\
\text { individuelle }\end{array}$ & & & \\
\hline $\begin{array}{l}\text { Rationalisation } \\
\text { budgétaire }\end{array}$ & & & \\
\hline $\begin{array}{l}\text { Communication } \\
\text { Simplification } \\
\text { administrative }\end{array}$ & & & \\
\hline
\end{tabular}

Les 6 premières lignes de ce tableau représentent les arguments traditionnels développés par les économistes et politistes anglo-saxons à propos des objectifs assignés au chèque. On remarque que les acteurs français citent systématiquement et spontanément au moins 3 arguments en décrivant un dispositif par chèque. Ces arguments sont évidemment formulés différemment en fonction du type de chèque décrit (ainsi, il n'est pas illogique que la motivation de remplacer une aide sociale obligatoire par un chèque soit $\mathrm{d}^{\prime}$ abord financière et ait pour but une limitation $\mathrm{du}$ choix $\mathrm{du}$ bénéficiaire plutôt que l'ouverture d'un secteur à la concurrence).

Les deux dernières lignes du tableau sont les autres arguments que nous avons pu identifier chez les acteurs et qui apparaissent nettement moins développés dans la littérature. 
Il apparaît donc que les chèques français et les chèques anglo-saxons sont non seulement des outils semblables, mais sont définis par les mêmes qualificatifs par ceux chargés de les mettre en place et de les distribuer à la population.

Il s'agit du principal résultat de notre recherche : non seulement le fonctionnement des chèques est le même, mais les représentations que s'en font les acteurs le sont également.

La principale question à laquelle nous avons ensuite dû répondre fut celle de la diffusion du chèque : comment un outil libéral, porteur du sceau du Nouveau Management Public a pu se diffuser au sein des collectivités françaises sans que jamais ses attributs ne soient démasqués ?

Si le chèque s'est diffusé en France à la suite du lancement du Chèque culture Rhône-Alpes en 1994, c'est parce que cet outil a su prendre des formes diverses et acquérir une souplesse idéologique lui permettant d'être revendiqué à la fois comme un outil libéral notamment par Charles Millon et comme un outil favorisant l'autonomie et la justice sociale par ses successeurs issus du Parti Socialiste.

Cette souplesse et cette nature protéiforme du chèque lui ont donc permis d'avancer masqué et d'apparaître comme un outil indispensable, créant même parfois une dépendance au chemin comme nous avons pu l'étudier avec la carte M'RA du Conseil Régional Rhône-Alpes (Lacheret, 2014).

Plusieurs autres arguments autour du chèque ont ainsi pu être développés, en faisant un objet aux multiples facettes.

\subsubsection{Le chèque comme vecteur de communication}

Le fait que la filiation néolibérale n'ait pas été apparente et revendiquée dans le discours de la plupart des acteurs a constitué le cœur de l'étude, une partie du problème à résoudre.

Certes, les arguments du libre-choix, de la rationalisation budgétaire, de l'encadrement de la dépense individuelle, du remplacement d'un programme existant et de la dépense sociale " acceptable " par le "client contribuable ", se sont retrouvés lors des entretiens avec les acteurs administratifs et politiques du chèque. La plupart du temps toutefois, le premier argument développé par ces derniers est la visibilité, la matérialisation, la tangibilité de l'aide individuelle.

Le chèque dans les collectivités françaises est donc d'abord considéré par les acteurs interrogés comme un outil de communication, et cela même s'il est utilisé pour répondre à des besoins sociaux comme le versement de l'APA, de la Prestation de Compensation du Handicap ou du complément au Revenu de Solidarités Actives.

Cet aspect n'apparaît quasiment pas dans les études anglo-saxonnes et le fait que l'aide soit matérialisée est, chez les chercheurs ayant étudié le "voucher » outre-Atlantique, surtout un attribut permettant de mieux en contrôler l'usage. La différence essentielle entre le chèque français et le " voucher » anglo-saxon tient donc dans sa représentation par les acteurs. C'est une des découvertes fondamentales de cette étude et un des facteurs qui expliquent le mieux la diffusion de cet outil.

Les causes de cette apparition du thème de la communication sont multiples et la première d'entre elles tient au fait que les prestataires privés commercialisant ces dispositifs ont mis en avant cet aspect dans leurs argumentaires de vente. 
Cette différence importante dans la représentation du chèque d'un côté et de l'autre de l'Atlantique ne modifie cependant ni la nature du chèque, ni le reste de l'argumentation des acteurs. II s'agit plutôt d'un camouflage idéologique qui a permis à cet outil d'être accepté et apprécié.

Cette rhétorique autour de la communication n'est en effet apparue qu'après le lancement des premiers chèques au sein des collectivités françaises. Les pionniers du Chèque culture Rhône-Alpes ne mettent en effet pas vraiment en avant la notion de visibilité de l'aide et revendiquent leur discours néolibéral lorsqu'ils exposent leurs représentations du chèque.

Le fait que le Conseil Régional Rhône-Alpes reconnaisse officiellement la filiation entre le Chèque culture et son successeur, la carte M'RA nous a également bien montré que si l'argumentaire avait évolué, l'objet était resté le même.

\subsubsection{Une différenciation en fonction de la nature du chèque}

L'un des autres axes de cette recherche fut de déterminer si les chèques de nature sociale et les chèques permettant de verser des aides facultatives à des individus ciblés comme peuvent l'être les chèques culture étaient de la même nature. Nous avons d'abord établi que ces dispositifs n'étaient pas apparus en même temps et n'avaient pas suivi des évolutions similaires.

Nous avons ainsi vérifié l'hypothèse selon laquelle les chèques permettant de verser des aides individuelles facultatives de type "chèque culture " étaient issus du modèle rhônalpin élaboré en 1993 et lancé en 1994 tandis que les chèques de type sociaux étaient apparus grâce à une impulsion de l'Etat avec la loi instituant le Chèque Emploi Service Universel et permettant de payer des aides sociales légales à l'aide de cet outil.

Nous avons d'ailleurs pu observer que la loi créant l'Allocation Personnalisée d'Autonomie en 2001, puis le constat que la montée en puissance de ce dispositif grevait largement les finances des Conseils Généraux, avaient conduit le Gouvernement à lancer le CESU dont le principal argument justifiant sa mise en place comme moyen de paiement au sein des départements est justement d'ordre financier.

Cette double naissance, inspirée de dispositifs anglo-saxons répliqués au niveau local d'un côté et matérialisée par une loi de l'autre, fut ainsi une réelle source de complication au cours de l'étude : s'agissait-il de la même chose et pouvait-on rattacher des objets en apparence si différents ?

Partant de cette interrogation, la recherche nous a permis dedémontrer que les chèques sociaux encadrés par la loi et les chèques établis "sur mesure " par les collectivités sont bien tous deux les " enfants » des "vouchers " anglo-saxons. L'enquête de terrain a permis de vérifier que, malgré leurs différences de formes et leurs genèses dissemblables, leurs natures étaient les mêmes et qu'il fallait les considérer comme des objets identiques

Les acteurs ont ainsi développé des arguments proches pour décrire les deux dispositifs. Les prestataires de services commercialisant ces chèques utilisent également des argumentaires de vente assez semblables, ce qui a permis de confirmer le raisonnement théorique : bien que leurs modalités d'arrivée sur le territoire national soient fondamentalement différentes, les chèques français de tous types correspondent à la définition du "voucher » et sont donc la réplication d'outils néolibéraux largement étudiés et répandus outre-Atlantique. 


\section{Le chèque, un outil plutôt qu'un instrument}

L'ambition de notre étude était de faire découvrir un outil du Nouveau Management Public servant au ciblage des aides que tout le monde manipule mais dont peu de chercheurs, en France, ont mesuré le sens politique réel. Pour cela, il était nécessaire de disposer d'un cadre de référence pour étudier les différents types de chèques utilisés par les collectivités territoriales.

Si l'approche par les instruments semblait être plutôt intéressante pour étudier le chèque dans le sens où ces objets constituent des dispositifs techniques à vocation générique permettant notamment de réguler le versement d'une aide individuelle et qu'ils sont neutres politiquement du moins en apparence, le risque d'utiliser une telle notion et la crainte d'un procès en " empirisme " nous ont fait préférer la notion d'outil, qui sied tout particulièrement au chèque.

Lester Salamon, dont nous avons étudié les travaux sur ce concept, cite en effet systématiquement le chèque au rang des outils. Ces derniers, pour lui ne sont que des dispositifs au service du politique pouvant être utilisés indifféremment sans pour autant dénaturer la politique publique qu'ils sont censés servir (Salamon, 2002).

Le chèque est donc un élément d'une "boîte à outil " du Nouveau Management Public qui est à la disposition du politique. Dans sa très longue liste d'outils, Salamon, cite notamment les crédits d'impôts, les avances remboursables, les prêts à taux préférentiels et, bien sûr, les chèques.

Cette notion d'outil est avantageuse dans le cadre d'une recherche en politiques publiques car elle est à la fois très large et relativement bien bornée. Les acteurs interrogés lors de l'étude définissent d'ailleurs le chèque comme un outil (bien évidemment, il ne s'agit que d'un mot et pas d'une référence à la notion définie par Salamon) et s'efforcent de le dépouiller de tout sens politique, ce qui est le but recherché par les utilisateurs de ce concept outre-Atlantique, qui souhaitent dépolitiser l'outil pour ne donner de sens qu'à la politique mise en œuvre par ce moyen.

Le chèque tel que nous l'avons étudié remplit parfaitement cette fonction. Nous sommes ainsi parvenu à définir empiriquement ce que pouvait représenter un "outil» dans le contexte des collectivités françaises.

Or, la conceptualisation de la notion d'outil de politiques publiques par des chercheurs américains rend plus neutre des concepts largement connotés comme le chèque. Son invention remonte, nous l'avons vu, à Milton Friedman, qui en faisait un élément essentiel de libéralisation et de démantèlement des monopoles publics en introduisant la notion essentielle de libre-choix parmi les bénéficiaires d'une aide individuelle.

Ce n'est qu'au cours des années 1990, lorsque le chèque est devenu un marqueur politique trop important aux Etats-Unis, qu'on s'est mis à le qualifier d'outil et qu'il a été inséré au milieu de nombreux autres moyens de paiement d'une aide individuelle.

C'est donc grâce à cette forme édulcorée " d'outil ", la recherche menée l'a amplement démontré, qu'il est arrivé en France et a pu se diffuser au sein des politiques publiques des collectivités territoriales.

\section{Le chèque et les administrations locales}


Notre postulat pour cette étude est qu'au sein de collectivités importantes comme peuvent l'être les régions et les départements, le poids des services administratifs est important dans la prise de décision.

L'une des conclusions était que l'administration avait un véritable rôle dans la décision et qu'elle pouvait à la fois être à l'initiative de politiques nouvelles tout comme elle pouvait contribuer fortement à l'échec de politiques publiques si elles ne convenaient pas à certains agents, pas nécessairement placés très haut dans l'organigramme.

Plutôt que de concentrer les entretiens sur des personnalités politiques, des membres de cabinet ou des directeurs généraux, il nous a semblé plus utile de partir de la base de la pyramide administrative. Ce raisonnement n'est pas souvent celui qui a cours dans la plupart des études de science politique où les chercheurs estiment sans doute plus important de commencer l'enquête de terrain par le sommet de la hiérarchie politique ou administrative. Ce qui est valable pour des études européennes et à l'échelle étatique ne nous semblait peut-être pas la meilleure solution ici.

Nous nous sommes également appuyés sur l'observation du fonctionnement des entreprises commercialisant les chèques pour estimer que les acteurs les plus proches du dispositif seraient sans doute ceux qui apporteraient le plus à l'étude.

Le chèque peut donc constituer, pour les fonctionnaires en charge de son traitement administratif, un véritable vecteur de changement et de promotion au sein de la collectivité. Notre recherche a en effet démontré que le chèque permet non seulement de tangibiliser l'aide en jouant le rôle de vecteur de communication, mais qu'il permet également de mettre le service thématique qui en assure la gestion au centre de l'organisation.

Ainsi, les agents assurant la gestion quotidienne d'un dispositif par chèque ressentent la plupart du temps une véritable fierté, une reconnaissance alliée au sentiment de se retrouver au centre du jeu. Lorsque le chèque couvre une thématique jusqu'à lors peu mise en valeur au sein de la collectivité, cette mise en avant est particulièrement spectaculaire et permet aux agents de voir augmenter leur poids symbolique au sein de la collectivité.

Cette appropriation du chèque par les fonctionnaires peut également avoir la conséquence inverse : la greffe peut ne pas prendre et le service peut tout à fait rejeter le chèque, allant jusqu'à en souhaiter la disparition et œuvrant en ce sens.

La souplesse de cet outil lui permet donc, et c'est un autre des résultats de cette recherche, d'être saisi et utilisé à des fins de promotion interne au sein de l'organisation par une partie de l'administration.

Le ciblage des populations en France dans le cadre des aides culturelles et sociales individuelles concerne énormément les collectivités qui ont soit pris l'initiative de la création de telles aides soit se les sont vues confier par l'Etat dans le cadre de transferts de compétences. Plusieurs facteurs ont poussé les collectivités territoriales à adopter les outils du ciblage que sont les chèques ou " vouchers".

L'ambition de l'étude a résidé sur un problème : il était difficile d'expliquer la diffusion des chèques dans l'ensemble des collectivités territoriales françaises.

Une fois qu'il a été démontré que le chèque était en lui-même un outil «inventé » par Milton Friedman et présenté comme un dispositif néolibéral bien qu'étant classé dans la catégorie des 
" outils» du Nouveau Management Public neutres politiquement, il s'agissait de comprendre pourquoi des collectivités territoriales françaises dont les exécutifs, plutôt classés à gauche de l'échiquier politique, créaient des programmes à base de chèques.

Une fois déterminés les arguments principaux utilisés par les analystes du chèque, il a fallu les comparer avec le contenu du discours des acteurs, au cours d'une étude ayant mobilisé 45 entretiens qualitatifs, et donc démontrer que si les chèques français avaient l'apparence de "voucher ", les acteurs utilisaient les mêmes termes pour en décrire l'utilité. Evidemment, tous les discours ne sont pas identiques et varient, nous avons pu le voir tout au long de l'étude, en fonction du type de chèque et du niveau où les acteurs se situent dans la collectivité.

Une telle expansion d'un instrument si connoté peut donc s'expliquer par une conjonction d'éléments qui ont visé à le vider de sa substance idéologique : la notion de ciblage, de "targeting", mêlée à l'introduction du " social care " a permis de camoufler l'aspect très libéral du chèque en en faisant un outil qualifié d'efficace et de pragmatique. Cependant, l'étude a montré que l'argument le plus marquant et ayant permis de faire accepter un outil de contrôle social et de ciblage aussi contraignant est celui de la communication. La transformation d'instruments connotés en outils de communication visant à promouvoir l'action d'une collectivité et de son président a permis leur introduction et leur multiplication dans un silence idéologique surprenant compte-tenu des débats ayant eu lieu outre-Atlantique sur le sujet, notamment du " school voucher ».

Si le néolibéralisme apparait en France par le biais d'instruments tels le ciblage des aides et les chèques, son arrivée et sa diffusion a ainsi nécessité un changement fondamental de discours, permettant de le faire accepter. 


\section{Références Bibliographiques :}

ACEMOGLU D., JOHNSON S., ROBINSON J., 2001, « The colonial origins of Comparative Development: an empirical investigation ", The American EconomicReview, 91, 5

ANAND S., KANBUR R., 1991, " Public Policy and Basic Needs Provision: Intervention and Achievement in Sri Lanka ",in DREZE J., SEN A (ed), The Political Economy of Hunger, Oxford Clarendon Press

BIBI S., DUCLOS J., 2007, "Equity and policy effectiveness with imperfect Targeting ", Journal of development economics, 83, pp. 109-140

BRADFORD D., SHAPIRO D., 1999, The economics of vouchers, National Bureau of Economic Research, Working Paper 7092

CAIN E., 2009, Social Protection and Vulnerability, Risk and Exclusion across the Life-Cycle, HelpAge International

CALDES N., COADY D., MALUCCIO J., 2004, "The cost of poverty transfer programs: a comparative analysis of three programs in Latin America», International Food Policy Research Institute, FCND,174

CHRISTENSEN., T., LAEGREID P., 2001, New Public Management : The Transformation of Ideas and Practice, Aldershot, Ashgate

COADY D., GROSH M., HODDINOT J., 2004, Targeting of Transfers in Developing countries: Review of Lessons and Experience, World Bank Research Institute

COMIA G., STEWART F., 1995, "Two errors of targeting ", in VAN DE WALLE D., NEAD K.(ed.), Public Spending and the Poor: Theory and Evidence, Johns Hopkins University Press

ESPING-ANDERSEN G., 1990, The Three Worlds of Welfare Capitalism, Princeton University Press

ESPING-ANDERSEN G., 1999, The Social Foundations of Postindustrial Economies, Oxford University Press

ESPING-ANDERSEN G., 2008, Trois leçons sur l'État Providence, Seuil

FRIEDMAN M., 1962, Capitalism and freedom, University of Chicago Press

GARATE E., 2010, La "Révolution économique » au Chili. A la recherche de l'utopie néo-conservatrice (1973-2003), Thèse de Doctorat en Histoire, EHESS

GELBACH J., PRITCHETT L., 2002, " More for the Poor is Less for the Poor - the politics of meanstested targeting ", The Berkeley journal of economic analysis and policy

GROSH M., 1994, Administering Targeted Social Programs in Latin America: From Platitudes to Practice, World Bank

HOOD C., 1991 « A public management for all seasons? », Public Administration, 69,1, pp. 3-19

HSIEH C., URQUIOLA Miguel., 2006, "The effects of generalized school choice on achievement and stratification: Evidence from Chile's voucher program ", Journal of Public Economics, 90, pp. 14771503

KERVYN DE LETTENHOVE M., 2012, «Conditional Cash Transfers in Latin America - Impact, scope and limitations ", Reflets et perspectives de la vie économique, 51 
LACHERET A., 2013, "L'évaluation comme instrument d'effacement du sens politique : la controverse autour de l'évaluation des schools vouchers américain ", Revue Française d'Administration Publique, 148, pp. 923-937

LACHERET A., 2014, L'aide sociale par le chèque, genèse et mise en œuure des dispositifs sociaux et culturels ciblés des collectivités territoriales, Thèse de doctorat de science politique, Université de Grenoble

LASCOUMES P., LE GALES P., (ed.), 2004, Gouverner par les instruments, Paris, Presses de la Fondation nationale des sciences politiques

LAUGIER S., 2011, " Le care comme critique et comme féminisme », Travail, genre et sociétés, 26

LAUTIER B., 1998, "Pauvreté et politiques de la pauvreté au Mexique ", in Richard Poulin, Pierre Salama (ed.), L'insoutenable misère du monde : économie et sociologie de la pauvreté, éditions Vents d'Ouest

LAUTIER B., 2010, "Les politiques sociales à l'épreuve des réformes de marché ", Revue internationale de politique comparée, 17, pp. 23-55

LUNDSGAARD J., 2002, "Ouverture à la concurrence et efficacité des services à financement public ", Revue économique de I'OCDE, 35, p. 84

OCDE., 2008, "Protection sociale, lutte contre la pauvreté et croissance pro-pauvres ", Revue de I'OCDE sur le développement, 9, pp. 37-63

OSBORNE D., GAEBLER T., 1992, Reinventing Government: How the Entrepreneurial Spirit is Transforming the Public Sector, Addison Wesley

PASQUIER-DOUMER L., LAVALLEE E., OLIVIER A., ROBILLIARD A., 2009, " Cibler les politiques de lutte contre la pauvreté dans les pays en développement ? Un bilan des expériences ", Revue d'économie du développement, 23, pp. 5-50

SALAMON L., (ed.), 2002, The Tools of Government: A Guide to the New Governance, Oxford University Press

SKOPCOL T., 1991, "Targeting Within Universalism: Politically Viable Policies to Combat Poverty in the United States», in JENCKS C., PETERSON P., (ed.), The Urban Underclass, Brookings Institution

SMITH J., SUBBARAO K., 2003, What Role for Safety Nets in Very Low Income Countries?, World Bank

STEUERLE E., (ed.), 2000, Vouchers and the provision of public services, Brookings Institute

TITMUSS R., 1958, Essays on the Welfare State, Yale University Press

TUCK L., LINDERT K., 1996, « From Universal Food Subsidies to a Self-Targeted Program: A Case Study in Tunisian Reform », World Bank Discussion Paper, 351

VAN DE WALLE D., 1998, "Targeting revisited», The World Bank Research Observer, 13, pp. 231-248

VAN KERSBERGER K., MANOW P., 2008, " The welfare state " inCARAMANI D., (ed.), Comparative politics, Oxford University Press, pp.520-545 